\title{
Qualitative Neuro Linguistic Programming using Meta Modeling and Python
}

\author{
Arun Kumar \\ AP, Research Scholar \\ FET, CSE, MRIIRS
}

\author{
Supriya P. Panda, PhD \\ Professor, HOD \\ FET, CSE, MRIIRS
}

\begin{abstract}
Neuro-Linguistic Programming (NLP) is a way to change the behavioral patterns of the human mind. This technique is very useful to reduce the stress and anxiety patterns of the mind. These patterns are considered as negative sentiments of the human mind. The sentiments and emotions will become the programming of the human mind. The human brain tends to store each piece of information and make it programmed in a short interval of time. NLP is a vast area to recognize the emotional as well as practical aspects of the human mind. NLP Workshops and various training methods have been used to change and trained the behavioral patterns of the mind. $\mathrm{V} / \mathrm{KD}$ used in earlier research and Meta-modeling in current research will be the important tools to convert the old patterns into new ones. This research paper will focus on the realistic mind pattern and improve it based on the current patterns of mind. NLP workshops and Qualitative surveys have been used in the methodology of the workshop. Data has been collected from various social communities and professional organizations. This research has been more impactful when used Meta modeling tools and Python for implementation. Meta-modeling tools are used to delete the old patterns of mind and create a new one as per the needs and requirements. A large number of participants attended the trial versions of NLP and enrolls themselves in NLP workshops to build new behavioral patterns. In this research, various NLP tools have been used to build new behavioral patterns of the human mind.
\end{abstract}

\section{Keywords}

Neuro-Linguistic Programming, Meta- Modeling Tools, Qualitative Surveys, Sentiment Detection, Sentiment Correction, Python

\section{INTRODUCTION}

NLP was invented by Dr. Richard Bandler in the 1970s. Dr. Bandler and his team worked on conventional physiotherapy techniques and discovered a novel approach to develop a new behavioral pattern system called Neuro-Linguistic Programming (NLP). NLP is a technique that can change the behavioral pattern of the human mind. This technique is used by various social communities and professional organizations. This technique creates a tremendous effect on the human mind, which can delete the old patterns and create new ones [2]. These patterns will be created based on external as well as internal environment. NLP used various tools like reframing, anchoring, $\mathrm{V} / \mathrm{KD}$, and meta-programming models. This paper focuses on various Meta- programming tools and their components used in neuro-linguistic programming process. Meta- models and tools are useful in various NLP workshops to implement the new behavioral patterns among millennial. The figure1 shows the communication model of NLP which established internal and external representation with the help of Meta programs [3]. NLP trainers use Meta -programming tools to establish effective communication in various workshops and training programs.

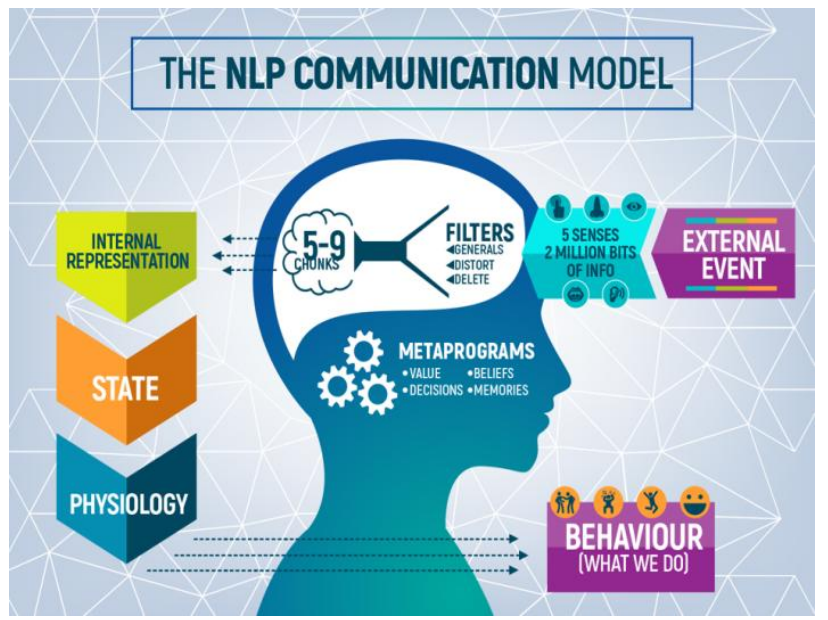

Figure 1: NLP Communication Model: Internet Source

\section{META MODELING in NLP}

Meta-modeling in NLP is the process to gather information about various behavioral patterns of the human mind. This process used deletion, distortion, and generalization as a tool of NLP to create a new thought pattern by changing the behavior of the human mind. Meta- programming is a way to train the human mind to perceive the information from the outside environment and convert it into the inner world through master senses.

Neuro-linguistic programming (NLP) is a way to implement new patterns and change the behavioral system of the human mind [5]. The human mind is gathering information about new ideas, thoughts, and various patterns. The human brain can be developed through external as well as internal patterns. These patterns can be changed in many ways while living from one way to another. Figure 2 shows how the information will be filtered after applying Meta modeling tools. NLP model is structured to communicate between the internal structure and various external events [4]. NLP model establishes the communication based on the current as well as old patterns of the mind. The filtration process will be based on the tools and training methods applied through NLP workshops. 


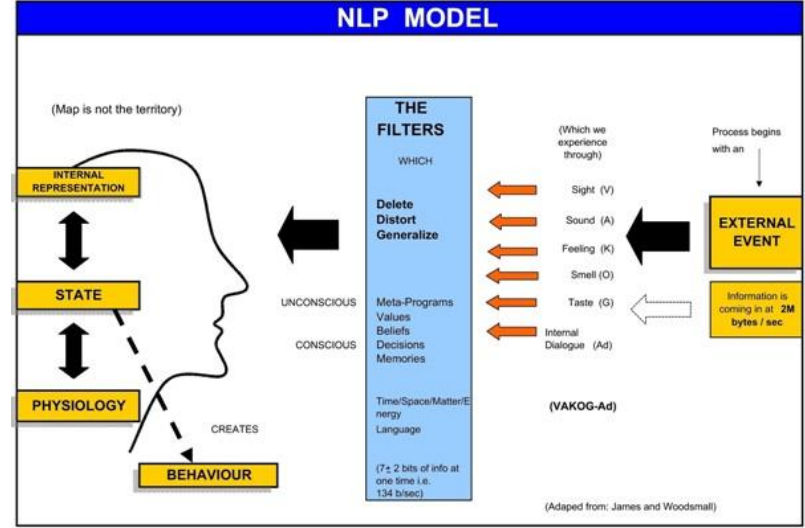

Figure 2: NLP Communication Model: Internet Source

\section{META MODELING TOOLS}

In Neuro-Linguistic Programming, every pattern is modeled with the use of Meta Modeling tools. There are three important tools used in Meta Modeling used in NLP workshops [8]. Also, these tools can help to build new behavioral patterns of mind. Deletion, Distortion, and Generalization are being used as a tool in Meta Modeling process.

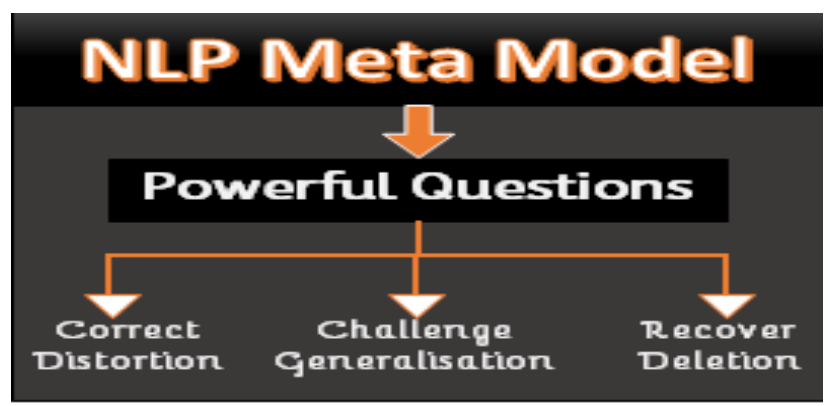

Figure 3: Meta Modeling Tools

\section{WORKING OF META MODELING TOOLS}

There are mainly four important tools used in the working of Meta modeling. These tools are used in the NLP workshops and various training methods. Following steps have been used to implement the process.

\subsection{Deletion Process}

The human brain tends to collect and save a vast amount of information. This information can be sorted according to the changing behavioral patterns of the human mind. The brain contains trillions of neurons and working through our master senses [10]. The master senses are working at a rate of 2.3 million per second whereas the brain gets this information at a rate of 134 bit per second. So when our brain is focused on one single point, a huge amount of information can be deleted. Thus, the deletion process in NLP plays an important role in terms of developing new behavioral patterns. NLP trainers work through various personal interviews and workshops on those patterns [6]. Theses workshops can be useful to know the realistic scenario and helps in developing the new patterns according to the various challenging environments.

\section{Procedure}

Step 1: The process starts with the personal interview of the participant of the workshop. After that, participants go into the session with questions and answers and have been asked about different aspects of their lives. With this procedure, the trainer gets all the information about the participant's behavioral patterns and their experiences of life. This process will collect all the information about the participant's patterns and experiences.

Step 2: Participants involve in the next step and go into the preparation process. According to new trends, this teaching pattern applies to designing the human mind. Participants have been passing through different old patterns that can be used to build the new ones and they can be eliminated by the instructions used in the testing phase after that.

Step 3: A parameter chosen by the NLP trainer is included in this phase. These parameters are based on the emotions of the person, previous thoughts, and the present states of their minds. Based on these criteria, NLP strategies can be applied to alter the behaviors of the mind.

Step 4: The last step of the deletion process in which trainers give instructions to each participant is based on various NLP techniques. These techniques will involve them to observe the current states of their mind. This method would eliminate all the unused mind patterns and make some room for new patterns to be formed.

\subsection{Distortion Process}

This tool of Meta- programming is used when there is a lack of understanding between the external and internal factors of the human mind [12]. It relates to the events that look like originally different from their real identity.

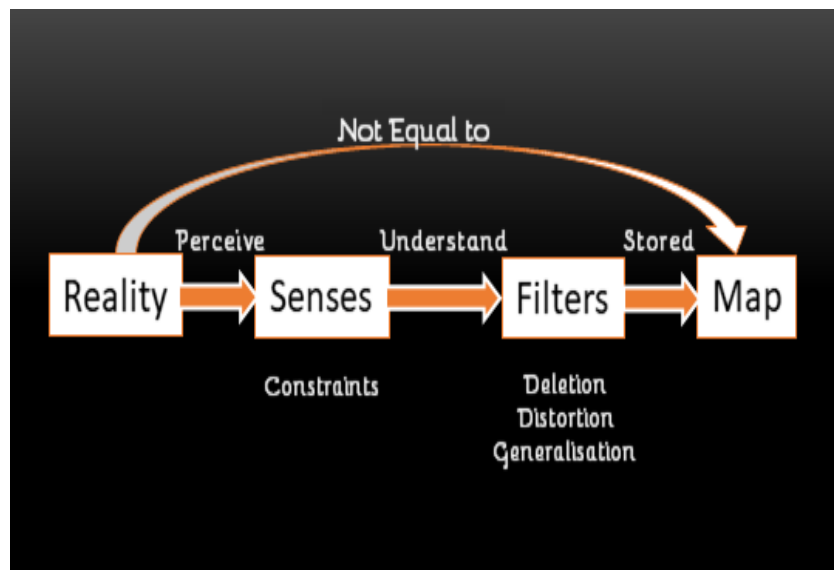

Figure 4: Meta Modeling Process \# 1

\section{Procedure}

Step 1: This step involves the mind-reading of the participants of the NLP workshops. Participants of the workshop undergo training in which they have been asked to express the real emotions and feelings related to their past life events or the current state of mind. They have been asked to observe their mind-tricks and their manipulations.

Step 2: The next step of the process involves "Named the emotion or Feelings of Expressions;" participants have been asked to name the emotional factor of all their feelings whether they are good or bad according to their perceptions of the mind. In NLP, this process is called lost per formative.

Step 3: After finding the result of the emotional factor, trainers can find the cause of the emotional factor. Human brain neurological patterns are capable of finding the reason behind every event. NLP provides the proper solution to process theses reasons by using various training programs.

Step 4: This step will be focused on the complex equivalence pattern where situations, ideas, and events produced different 
meanings about realistic patterns. To improve these types of patterns, NLP trainers used complex equivalence techniques to balance the emotional factors according to the external and internal environment.

\subsection{Generalization Process}

Generalization is the last step of the Meta modeling technique. It converts the information as exact as it will be. It means that there is no modification in the realistic process. Our mind tends to store a huge amount of information [9]. When you ask a person to recall the oldest information of his childhood then chances will be less to express the whole event. It is due to the huge amount of data stored in our conscious mind database. So generalization helps to delete unused data from the mind and focus on the recall ones. Generalization filters the data and converts it as per the situation demand. The following steps are involved in the NLP workshop while using the generalization process.

\section{Procedure}

Step 1: This step involves data collection from various sources of mind through qualitative surveys. Through these surveys, the NLP trainer's process participants mind and collect all the logic and facts that will be useful to retrain the mind and its psychology.

Step 2: After data collection, this process involves the situations that can be related to the information from the past or it can be from the current situation. Situations are dependent on the internal structure of the mind. The pretrained pattern of the mind can convert the situation into good or bad parameters.

Step 3: After knowing the current state of the participants' mind NLP trainer will be applicable to apply the NLP techniques. NLP techniques are different for each individual and depend upon the pre-trained pattern of their mind.

Step 4: The last step of generalization involves the filtering of data by applying NLP techniques. This process will filter data and divide $\mathrm{s}$ per the trained pattern of mind. This will fetch the exact information from the mind and enable it to train it according to the internal and external environments.

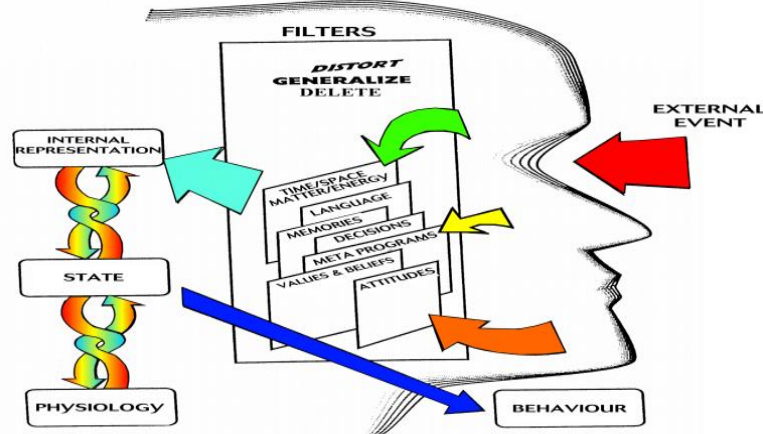

Figure 5: Meta Modeling Process \# 2

Figure 4 explains the working of the Meta Modeling process. This figure shows the concept of how Meta Modeling tools filter unused information from the human mind and create space for new emerging patterns. The new emerging patterns will be based on the various perspectives used in personal and professional environments. The NLP workshops based on the requirements and needs of the participants, use Meta Modeling tools.

\section{NLP Communication Model}

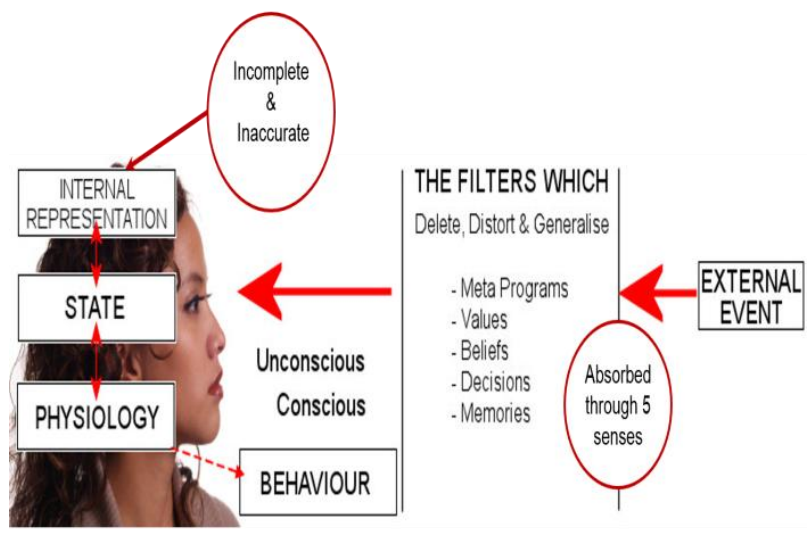

\section{Experience $\rightarrow$ Thoughts $\rightarrow$ Feelings $\rightarrow$ Actions}

Figure 6: Meta Modeling Process \# 3

This process generalization stored all the events related to the past as well as current situations of mind. Generalization in NLP is used to accept the realistic behavioral patterns mind and show it as original as they are.

\section{RESULTS OF NLP WORKSHOP}

Table 1: NLP Workshop Results

\begin{tabular}{|l|l|l|l|}
\hline NLP Testee & $\begin{array}{l}\text { NLP Training } \\
\text { Duration }\end{array}$ & $\begin{array}{l}\text { Negative } \\
\text { Sentiment } \\
\text { Detection }\end{array}$ & $\begin{array}{l}\text { Positive } \\
\text { Setiment } \\
\text { Detection }\end{array}$ \\
\hline Testee\#1 & $3-5$ & $66 \%$ & $34 \%$ \\
\hline Testee\#2 & $5-10$ & $45 \%$ & $55 \%$ \\
\hline Testee\#3 & $10-15$ & $30 \%$ & $70 \%$ \\
\hline Testee\#4 & $15-20$ & $25 \%$ & $75 \%$ \\
\hline Testee\#5 & $20-25$ & $20 \%$ & $80 \%$ \\
\hline Testee\#6 & $30-35$ & $15 \%$ & $85 \%$ \\
\hline Testee\#7 & $35-40$ & $10 \%$ & $90 \%$ \\
\hline
\end{tabular}

Table 2: NLP Workshop Results

\begin{tabular}{|l|l|l|l|}
\hline NLP Testee & $\begin{array}{l}\text { NLP } \\
\text { Training } \\
\text { Duration }\end{array}$ & $\begin{array}{l}\text { Actual } \\
\text { Negative } \\
\text { percentage }\end{array}$ & $\begin{array}{l}\text { Negative } \\
\text { Sentiment } \\
\text { Correction }\end{array}$ \\
\hline Testee\#1 & $3-5$ & $80 \%$ & $14 \%$ \\
\hline Testee\#2 & $5-10$ & $85 \%$ & $40 \%$ \\
\hline Testee\#3 & $10-15$ & $85 \%$ & $55 \%$ \\
\hline Testee\#4 & $15-20$ & $90 \%$ & $65 \%$ \\
\hline Testee\#5 & $20-25$ & $90 \%$ & $70 \%$ \\
\hline Testee\#6 & $30-35$ & $90 \%$ & $75 \%$ \\
\hline Testee\#7 & $35-40$ & $90 \%$ & $80 \%$ \\
\hline
\end{tabular}

The results of the NLP workshop and various training methods can be collected through the tabular form as shown in Table 1. This table is based on the negative and positive sentiment detection of participants. When the number of days of the training methods increased then the negative sentiment detection is decreased as shown in the Table 2. Here are the following points used in methodology. 


\section{METHODOLOGY}

There is a change in the behavioral habits of the human mind when applying training methods for about forty days. Here participants undergo training for 3-5 days. Their actual negative sentiment detection percentage was almost 80 percent and after applying Meta modeling tools there will be an improvement of 14 percent less detection in their negative sentiments. In the next step, the number of days for the workshop has been increased participants undergo training for 5-10 days. Their actual negative sentiment detection was almost 85 percent. After applying Meta modeling tools the improvement factor moves to approximately 40 percent. Then number of days increases at the interval of $[5,10,15,20,25,30,35,40]$. So, the improvement factor for the correction of negative sentiment is improved as the number of days of the teaching approaches has increased.

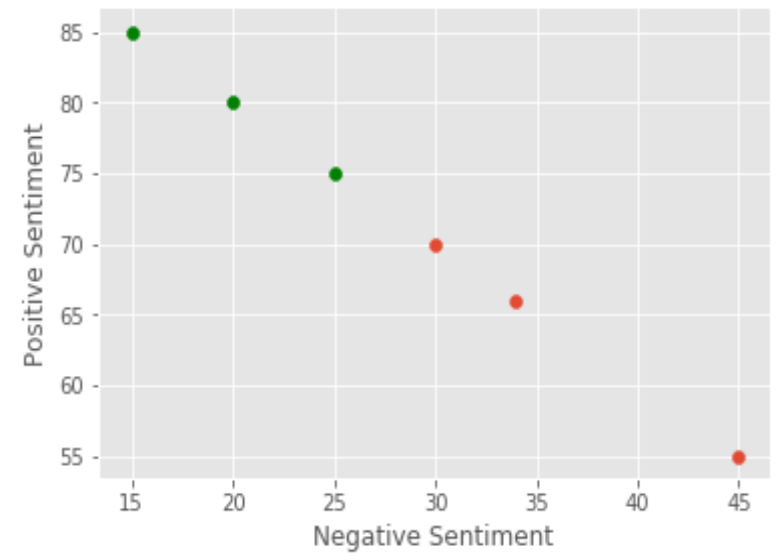

Figure 7: NLP Sentiment Detection Graph

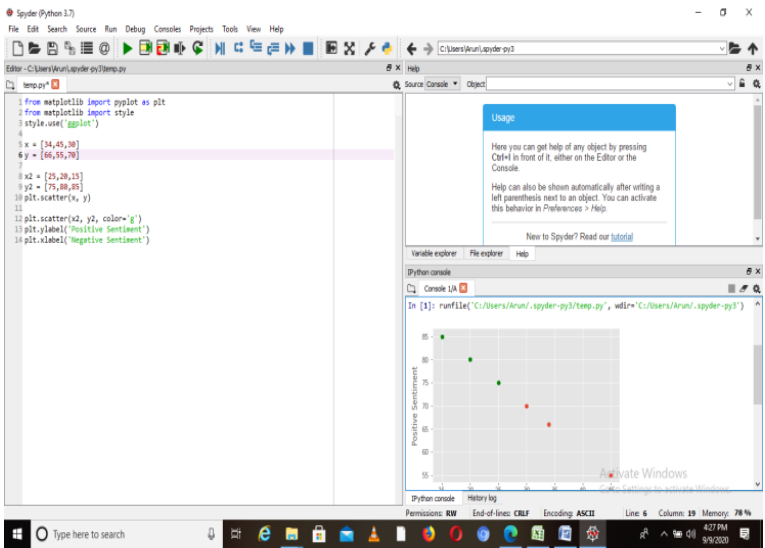

Figure 8: Spyder (Python 3.7) snippet

Figure 6 shows the negative sentiment correction of all the participants trained in NLP workshops. NLP trainer has set a limit to check and improve the performance factor of negative sentiments. The red dots in the graph shows the negative sentiment correction below 70 percent and the Green dots in the graph show the above 70 percent of negative sentiment correction. Also the python spyder (Python 3.7) is being used for the analysis of sentiment detection.

\section{CONCLUSION \& FUTURE SCOPE}

The NLP workshop process and its practices can produce a tremendous impact on the human mind and its behavioral patterns. These behavioral patterns always changed with the effect of internal and external environments. By applying Meta-modeling techniques through NLP, it is concluded that the human brain system always changed with the changing pattern based on the various situations and conditions. The human mind is conditioned with many social perspectives. These perspectives make behavioral patterns as per the requirements of mind. The mind tends to collect all these perspectives and graphed them in such a way that it can be programmed and stored in a memory system. These patterns can be used by the mind if there are useful and impactful for the current required scenario. This research used the Metamodeling tools to increase the performance factor of negative sentiment detection through the NLP workshop. The results are showing the improvement factor in behavioral patterns of mind as compared to the actual negative sentiment reported at the beginning of the workshop. Thus an increase in the number of days of practice and training will be an increase in the negative sentiment corrections. So, NLP workshops can change the behavioral pattern of the human mind when used with Meta- modeling tools as per the requirements of the external and internal environments i.e., endogenous and exogenous forces.

\section{REFERENCES}

[1] 1.Ahmad, K. Z., 2017, Improving Emotional Intelligence using Neuro Linguistic Programming techniques, International Conference on Advances in Business, Management and Law (ICABML), Vol. 1, No. 1, pp. 172-183.

[2] 2. Carey, J., Churches, R., Hutchinson, G., Jones, J., \& Tosey, P. ,2010. Neuro-Linguistic Programming and Learning: Teacher Case Studies on the Impact of NLP in Education, Online Submission.

[3] 3. Florina, G.E., Teodor, G.V., Cornelia, P.S. and Marin, D., 2015, Neuro-linguistic programming and the relationship between attention and anxiety in Alpine skiing juniors, Procedia-Social and Behavioral Sciences, 191, pp.1634-1638.

[4] 4. Gaspar, R., Pedro, C., Panagiotopoulos, P. and Seibt, B., 2016, Beyond positive or negative: Qualitative sentiment analysis of social media reactions to unexpected stressful events, Computers in Human Behavior, 56, pp.179-191.

[5] 5. Gibson, B. ,2011, The Complete Guide to Understanding and Using NLP: Neuro-linguistic Programming Explained Simply. Atlantic Publishing Company.

[6] 6. Grosu, E. F., Grosu, V. T., Preja, C. A., \& Iuliana, B. B. ,2014, Neuro-linguistic Programming based on the Concept of Modelling, Procedia-Social and Behavioral Sciences, 116, 3693-3699.

[7] 7. Grinder, J., \& Pucelik, F. ,2012, the Origins of Neuro Linguistic Programming, Crown House Publishing.

[8] Hosseinzadeh, E. and Baradaran, A., 2015, Investigating the Relationship between Iranian EFL Teachers' Autonomy and Their Neuro-Linguistic Programming, English Language Teaching, 8(7), pp.68-75.

[9] Karunaratne, M., 2010, Neuro-linguistic programming and application in treatment of phobias, Complementary therapies in clinical practice, 16(4), pp.203-207.

[10] Kök, İ., 2014, Listening comprehension achievement and brain dominance, Procedia-Social and Behavioral Sciences, 122, pp.329- 334. 
[11] Kotera, Y., \& Sweet, M. (2019), Comparative evaluation of neurolinguistic programming. British Journal of Guidance \& Counselling, 1-13.

[12] Lashkarian, A. , and Sayadian, S. ,2015, The effect of NeuroLinguistic Programming techniques on young Iranian EFL Learners' motivation, learning improvement, and on teacher's success, ProcediaSocial and Behavioral Sciences, 199, pp.510-516.

[13] Louis Sinclair. (2019), A Complete Guide to Neuro Linguistic Programming.

[14] Neidhardt, J., Rümmele, N. and Werthner, H., 2017, Predicting happiness: user interactions and sentiment analysis in an online travel forum, Information Technology \& Tourism, 17(1), pp.101-119.
[15] Learners' motivation, learning improvement, and on teacher's success. Procedia-Social and Behavioral Sciences, 199, pp.510-516.

[16] Neidhardt, J., Rümmele, N. and Werthner, H., 2017, predicting happiness: user interactions and sentiment analysis in an online travel forum, Information Technology \& Tourism, 17(1), pp.101-119.

[17] Passmore, J. and Rowson, T., 2019, Neuro-linguistic programming: A review of NLP research and the application of NLP in coaching. International Coaching Psychology Review, 14(1), p.57.

[18] Roderique-Davies, G., 2009, Neuro-linguistic programming: cargo cult psychology, Journal of applied research in higher education, 1(2), pp.58-63. 